\title{
Perfiles de delincuentes especializados y delincuentes versátiles estudio cuantitativo basados en la información del ISRD-2 ${ }^{1}$ Suiza
}

\author{
Specialized and versatile criminal profiles: \\ quantitative study based on information from Swiss ISRD -2 \\ ZEINAB OBEIDA \\ Magister en Derecho y Ciencias Penales. Mención Criminologia y seguridad \\ Universidad de Lausanne Suiza \\ Etablissements pénitenciers de la Plaine de l'Orbe \\ zeinab.obeida@gmail.com
}

Recibido: Septiembre 17 de 2012

Aceptado: Noviembre 20 de 2012

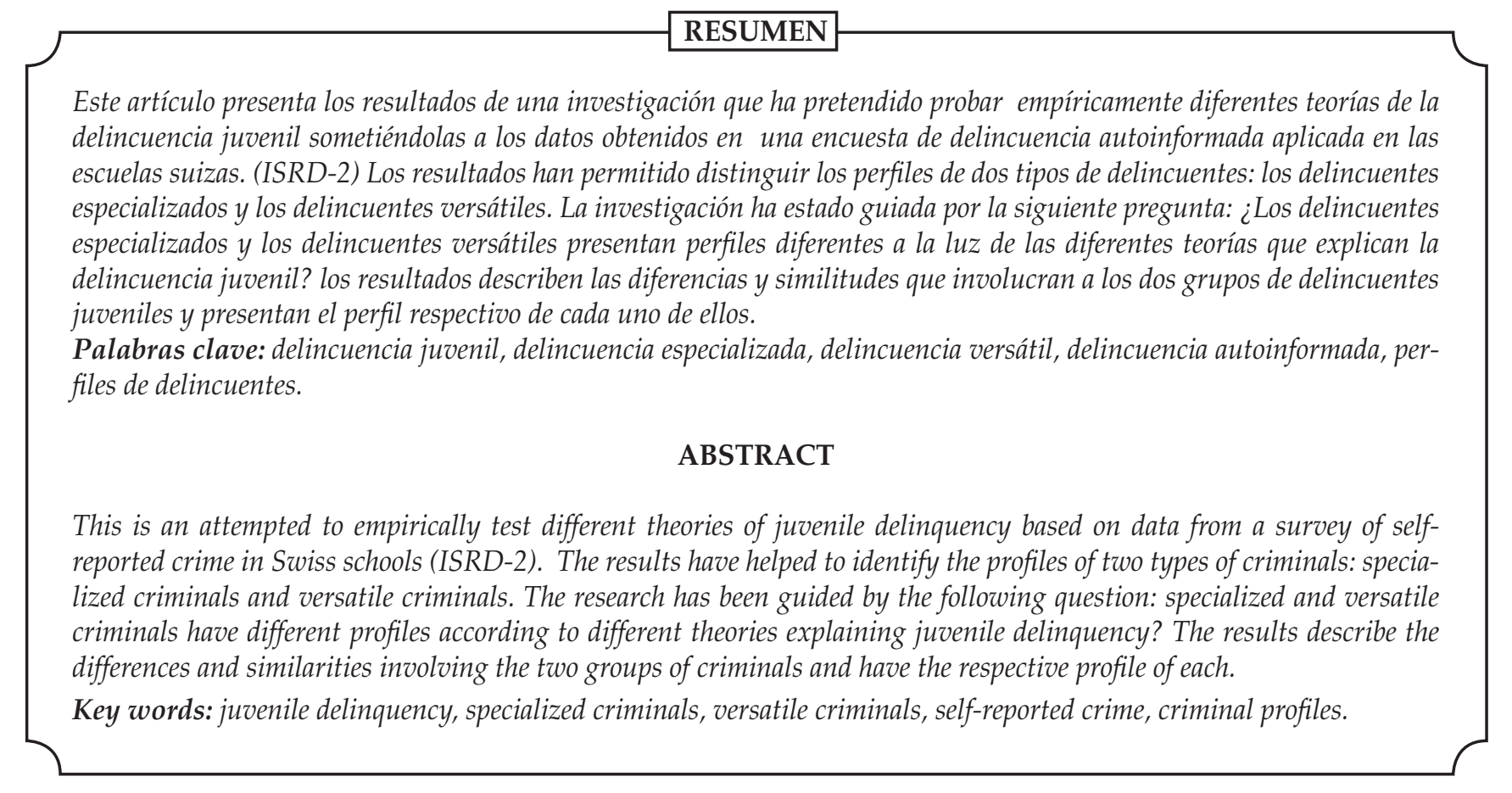

International Self-Reported Delinquency Study 2. 


\section{Introducción}

El estudio al cual se hará referencia a lo largo de este artículo, ha buscado someter al examen empírico diferentes teorías de la delincuencia juvenil confrontandolas con los datos de una encuesta de delincuencia autoinformada realizada entre jóvenes escolares suizos. Los resultados han permitido distinguir los perfiles de dos tipos de delincuentes: los delincuentes especializados y los delincuentes versátiles. Los primeros cometen un tipo reducido de delitos, mientras que los segundos cometen una variedad. La pregunta que ha guiado la investigación es la siguiente: ¿presentan los delincuentes especializados y los delincuentes versátiles diversos perfiles a la luz de las diferentes teorías que son abordadas en el marco teórico del estudio? Para responder a esta pregunta, se han sometido a prueba diversas hipótesis utilizando datos de la ISRD-2 una encuesta de delincuencia autoinformada, aplicada en varias escuelas de Suiza.

En este artículo se presenta en primer lugar, diversos enfoques teóricos que intentan explicar las diferentes características que rigen los dos grupos objeto de este estudio. En la sección de metodología se resumen las hipótesis que se han tratado de probar y la manera como se operacionalizaron adaptandolas a los datos de la encuesta ISRD-2. Finalmente, en la parte de los resultados se describen las diferencias y similitudes que hay entre los dos grupos de delincuentes juveniles ya mencionados y se describe el perfil respectivo de cada uno de ellos.

\section{Enfoques teóricos}

Las diversas teorías abordadas en el estudio se refieren a las diferencias que presentan los delincuentes especializados en comparación con los delincuentes versátiles. Así se tiene en primer lugar, las teorías del desarrollo, las cuales consideran que los factores que aparecen de manera precoz en la vida de una persona influyen el tipo de implicación de ésta en la delincuencia. Por su parte la teoría del autocontrol sirvió para abordar el problema en términos de la propensión a la delincuencia. Estos dos enfoques se consideraron conjuntamente para explicar cómo ciertos rasgos de personalidad conducen a diferentes formas de delincuencia. Igualmente se tuvo en cuenta las teorías situacionales para tratar la problematica en términos de los grupos de pares frecuentados por los jóvenes y estas teorías tratan de explicar cómo estas frecuentaciones conducen a una delincuencia especializada o a una polivalente. Por último, se concluyó con la teoría de la elección racional que define cómo la elección del estilo de vida y el entorno en el que está inmerso el individuo puede conducirlo a un determinado tipo de delincuencia o a otro.

\section{Las teorías del desarrollo}

La criminología del desarrollo propone varias clasificaciones empíricas de los delincuentes. Entre ellas, algunas toman como elemento principal de clasificación la edad de iniciación a la delincuencia, en Inglés "onsetage" (Gottfredson, 2005). La edad, es un buen factor predictor y explicativo de la implicación de un individuo en una actividad criminal. Otras clasificaciones se basan en hechos traumáticos acaecidos en la vida de una persona que pueden empujarla a una conducta desviada.

Varios estudios especialmente algunas investigaciones longitudinales (Moffitt, 1993, 1997, Tremblay, 2000; Farrington, 1986; Blumstein et al, 1986) muestran una fuerte correlación positiva entre la edad, la frecuencia y la diversidad de comisión de delitos. Sin embargo, estos mismos estudios con frecuencia suelen empujar el análisis en el sentido de considerar que no es la edad del joven en si misma la que predomina en las diversas medidas de la delincuencia, como la edad de comisión de los primeros actos delictivos (Piquero et al., 1999). Efectivamente, quienes se implican en conductas delictivas a una temprana edad tienden a cometer un número mayor de diveros delitos, en un período más largo y con una frecuencia mayor que los que se convierten en delincuentes a una edad más tardía. Hay aquí una dicotomía entre dos grupos de jóvenes, el uno compuesto por delincuentes llamados precoces y el otro de delincuentes llamados tardíos. (Monahan \& Piquero, 2009).

Moffitt (1993) presenta una taxonomía de la delincuencia que describe dos categorías de delincuentes juveniles. El primer grupo: individuos -alrededor de $4-10 \%$ de la población delincuente- en los cuales el comportamiento delictivo aparece temprano y persiste durante toda la vida. Esto seria el resultado de particulares rasgos de personalidad manifestados a una edad muy joven y tendría consecuencias a largo plazo a menudo dramáticas y persistentes, difíciles de modificar una vez instalados. Las características particulares de la personalidad que los investigadores (Piquero et al., 2000) señalan son rasgos tales como la irritabilidad, la impulsividad o un bajo nivel de auto-control. Un niño con tal personalidad se socializa, entonces, a menudo de manera disfuncional lo que disminuye sus posibilidades y oportunidades para desarrollar actitudes pro-sociales, ya sea hacía su grupo de pares o hacia cualquier otra persona de su entorno. Estas conductas inadecuadas, manifestadas a menudo en la infancia por un comportamiento problemático, genera reacciones aversivas en las distintas personas que interactúan con el niño, el cual se orientará muy temprano hacia conductas desviadas teñidas de agresividad, de violencia y en todo caso contrarias a las normas convencionales establecidas 
por la sociedad (Tremblay, 2000). Durante el crecimiento y desarrollo de estos individuos, estos rasgos particulares van a fortalecerse e interactuar con otros factores ambientales, familiares o sociales que causan en la mayoría de los casos comportamientos delictivos y la perpetración de actos violentos relativamente graves. Ellos también se implican en los tipos de delitos mucho más triviales, pero no se satisfacen. De tal forma que ellos cometen también delitos violentos mucho más grave, que consiste no sólo en daños a la propiedad, sino a menudo en lesiones a las personas. Esta delincuencia versátil se manifestará muy temprano debido a que el individuo se siente precozmente atraído por la conducta delincuente.

El segundo grupo de delincuentes descritos en los trabajos de Moffitt (1997), abarca jóvenes mucho mejor socializados, no presentando por lo general particulares rasgos de personalidad como no sea una relativa impulsividad, una preferencia por las recompensas a corto plazo o incluso una necesidad y búsqueda de sensaciones fuertes. Sus actos delictivos son considerados como una reacción de adaptación normal al finalizar la infancia. Los adolescentes pasan por diversos cambios biológicos y sociales, asi los cambios biológicos (tales como los niveles elevados de testosterona en los varones) podría aumentar el nivel de irritabilidad. Con respecto al cambio social, el hecho que los adolescentes se situen en una etapa intermedia entre la niñez y la edad adulta implica que ellos quieran tener acceso a los privilegios de los mayores, sin ser lo suficientementes maduros y responsables para poder beneficiarse. Esta diferencia provoca una reacción de rebeldía en este estado de transición que se manifiesta a veces por actos desviados o incluso delincuentes. Sin embargo, estos son en su mayoría pequeños actos, sin mucha violencia y limitados al corto período de la adolescencia. Estos delincuentes se especializan en delitos que simbolizan los privilegios de los adultos para demostrar su independencia frente al control parental (Guerette, Steinus y McGloin, 2005). Su gusto por la delincuencia aparece sólo durante esta crisis, los delitos no van a cometerse entonces que a una edad relativamente tardía.

\section{La teoría del autocontrol}

La teoría del autocontrol tal como ella es desarrollada en particular por Gottfredson yHirschi (Hirschi y Gottfredson, 1990; McGloin et al, 2007; Mason y Windle, 2002) describe dos tipos de controles. El primero es interno, es decir que el individuo tiene los recursos necesarios para tener un control sobre sus propias emociones, impulsos y deseos. Pero también puede tratarse de un control externo ejercido por los otros actores del entorno del jóven, especialmente en términos de supervisión parental, es decir, el hecho de que los padres conozcan y supervisen las actividades de sus hijos, sobre todo durante su tiempo libre. En el presente estudio el interes se centro unicamente en el control interno, llamado nivel de autocontrol.

De acuerdo con Gottfredson y Hirschi (1990), el nivel de autocontrol de un individuo tendría también un impacto significativo en la edad de inicio, la versatilidad, la frecuencia y la gravedad de la conducta delictiva del individuo. El bajo nivel de autocontrol, en inglés "self-control" mencionado en estos estudios se define por: el carácter impulsivo, egocéntrico de un individuo, la falta de perseverancia, la necesidad de estimulación buscando vivir emociones fuertes y la atracción por actividades de riesgo (Gottfredson y Hirschi, 1990).

Un bajo nivel de auto control engendra pues una multitud de problemas de conducta, incluyendo el hecho de participar en la delincuencia a muy temprana edad. La frecuencia asi como la diversidad de esta actividad aumenta con la edad (Mazerolle, Brame, Paternoster, Piquero y Dean, 2000). Este aumento se puede atribuir al hecho de que -en especial durante el período de la adolescencia durante el cual los jóvenes exploran un nuevo universo, muy rico y estimulante- el individuo adquiere más y más independencia asi como una nueva libertad sobre la elección de sus propias actividades. Desde un punto de vista estadístico, el nivel de auto-control y las oportunidades están fuertemente correlacionados. Por lo tanto, es común que las personas con bajo autocontrolar prefieran las situaciones criminogenas. Véase por ejemplos todas las situaciones en las que la persona es confrontada a la promiscuidad sexual, el consumo de alcohol o de drogas (Mazerolle et al., 2000). En resumen, el joven dispone de largos períodos de tiempo de ocio que le conducen a situaciones en las que una multitud de oportunidades de cometer todo tipo de crímenes tan diferentes unos de otros, está abierta para él.

El individuo se encuentra inmerso en un mundo donde las tentaciones son fuertes y muchas. Dada su impulsividad él no resistirá que muy difícilmente la atracción que le provoca la comisión de delitos. Por lo tanto, se dedicará a una delincuencia tan versátil como las oportunidades que se le presentan. Gottfredson y Hirschi (1990) plantean incluso que un bajo nivel de autocontrol está tan fuertemente correlacionado con la tasa de versatilidad de la delincuencia, que ésta sería un índicio válido para medir el nivel de auto-control.

En cuanto a la delincuencia especializada, un alto nivel de autocontrol representaría un obstáculo considerable para la comisión de delitos. A pesar de las muchas oportunidades que se ofrecen al individuo, este estaría en capacidad de controlarse a sí mismo y de resignarse a no pasar al 
acto si su único objetivo es satisfacer los impulsos internos. Además, estas personas tienen más tendencias de dirigirse hacia actividades estructuradas, legales y prosociales (Mazerolle et al., 2000).

\section{Propensión a la delincuencia}

Los defensores de la teoría de la propensión a la delincuencia argumentan que las causas de la variación en el comportamiento delictivo pueden observarse a la luz de las variaciones en ciertos rasgos de personalidad (Dean, Brame y Piquero, 1996).

Este enfoque tiene en cuenta tanto los elementos situacionales como los factores de desarrollo, ambientales y rasgos de personalidad para explicar el paso al acto delictivo. Las investigaciónes tratan de comprender de qué manera la tendencia de un individuo a cometer actos delictivos va influir sus actividades ordinarias y cómo ellas, influyen en la implicación de la persona a tomar una u otra dirección de comisión de delitos. Esta teoría establece un lazo entre la teoría puramente situacional, que concede una importancia predominante a las oportunidades, y las teorías del desarrollo centradas especialmente en los factores intrínsecos del individuo. La tendencia de una persona a la realización de actividades criminales, representa una forma de motivación que se extiende sobre un continuum y es relativamente estable. La principal medida utilizada por los investigadores (Gottfredson y Hirschi, 1990, 2003) es el grado de autocontrol del individuo. Aclarése sin embargo que no se trata de afirmar que la existencia de rasgos de personalidad predispone de manera absoluta a la criminalidad (Gottfredson y Hirschi 1989, 1993).

Las oportunidades son consideradas en el marco de esta teoría, como factores endógenos de motivación y esto por dos razones: la primera siendo un sesgo de selección, la segunda un sesgo de percepción. En primer lugar, los delincuentes con una baja propensión a la delincuencia son más especializados, pues seleccionan principalmente los eventos criminales que se les ofrecen. La diversidad en las conductas delictivas puede explicarse por un bajo nivel de autocontrol que causa dificultad para resistir la tentación de cometer un delito cuando el individuo se enfrenta a una oportunidad atractiva (Gottfredson, 2005). De otro lado, una alta propensión a la delincuencia tendrá igualmente una influencia en la percepción que el individuo tiene del mundo exterior. De hecho, este persivirá más las oportunidades de cometer delitos y prestara mayor atención a los detalles que le permitiran cometerlos y esto en cualquier situación mientras que un individuo con una baja propensión no considerara el mundo de esta manera. De hecho, él no percibe su entorno como principal terreno para las actividades delictivas.

\section{Teorías situacionales}

El enfoque de las actividades rutinarias (Cohen y Felson, 1979) trata de comprender cómo las actividades y la rutina diaria de un individuo puede llevarlo a una actividad criminal. Según Cohen y Felson (1979), un delito se produce cuando están presentes tres elementos: un autor motivado, un objetivo atractivo y la ausencia de un agente protector del objetivo. El estilo de vida adoptado por un joven influye en gran medida en su exposición al riesgo de cometer delitos, siendo los más expuestos aquellos que pasan mucho tiempo "andando de un lado a otro" con sus amigos en actividades no estructuradas y sin supervisión. De hecho, una persona joven dispone de mucho de tiempo libre que ella emplea de diferentes maneras.

En este estudio se buscó establecer que tipos de actividades ordinarias influyen la forma de criminalidad en la cual el individuo se implica y lo lleva ya sea a cometer una diversidad amplia de delitos o al contrario a especializarse en un tipo de delito bien focalizados. Entonces el entorno social y los eventos ocurridos en la vida de un individuo tendrían un impacto en la especialización y la diversidad de la delincuencia de éste (McGloin, Sullivan, Piquero, y Pratt, 2007). Sin embargo, la relación entre las actividades ordinarias o de rutinarias de una persona y la implicación de esta en una u otra tendencia de la criminalidad no es evidente para todos los autores. Los defensores de la teoría de las oportunidades, los cambios en las circunstancias de vida de un individuo engendrarían tambien cambios en su comportamiento criminal. En este estudio se sugiere aquí que son unicamente las situaciones sociales y ambientales que influyen de manera directa los comportamientos del delincuente, y esto excluyendo todas las otras razones. Por el contrario, los defensores de la propensión al crimen plantean que son las diferencias interindividuales que llevarían a cada delincuente hacia una $\mathrm{u}$ otras circunstancia de vida. Dicho de otra manera, es la propia persona la que se orientaría hacia actividades rutinarias propiciadoras de conducta delictivas y a adoptar un estilo de vida basado en la delincuencia. Ya se ha abordado la problemática bajo el angulo de la propensión al delito véase con más detalle cómo la teoría de la oportunidad intenta explicar la diversidad y la especialización de la delincuencia.

\section{Oportunidades y grupos}

La teoría cognitiva del potencial antisocial, postula entre otras cosas, que los cambios en el estilo de vida de una persona, tal como el hecho de casarse, encontrar un trabajo o cambiar de residencia generan también cambios en las actividades rutinarias de esa persona que pueden potencialmente reducir o aumentar el número y la natu- 
raleza de las oportunidades de comisión de delitos que se presentan a ella (Farrington, 2005).

En este estudio, la población de interés estuvo compuesta por los adolescentes en escolaridad obligatoria que tienen un estilo de vida más o menos ordinario, regido por horarios de clases. Si bien es cierto que los estudiantes disponen de su tiempo libre a su discreción, cambios importantes en su estilo de vida no dependen realmente de ellos, por ejemplo, ellos no tienen la edad suficiente para casarse, pueden dificilmente separarse de sus familias si no se comprende con esta, no pueden cambiar de escuela como ellos lo consideren conveniente (Armstrong, 2008). El estilo de vida que llevan esta relativamente condicionado por la situación de dependencia económica y legal con respecto a sus padres.

Sin embargo, fue posible aplicar esta teoría al estudio, analisando los grupo de amigos que frecuentan los jóvenes (Warr, 1996). Parece que efectivamente el grupo de amigos tendría un efecto directo no sólo en la cantidad sino también la calidad y la naturaleza de las oportunidades que se ofrecen al delincuente. En esta perspectiva el estudio, se guió por dos corrientes de pensamiento opuestas. Una proponiendo que los individuos de un mismo grupo se especializan en un tipo de delito, la otra que, postula que el hecho de pertenecer a un grupo engendra una delincuencia versátil.

En la primera propuesta, se sabe por la investigación llevada a cabo por Reiss (1986) que la mayoría de los jóvenes delincuentes cometen sus crímenes en grupos. La delincuencia en "solo" es relativamente rara entre los jóvenes y se produce muy excepcionalmente antes del fin de la adolescencia o acomienzo de la adultez. Estar en compañía de sus pares desviados se convierte para los jovenes infractores en un requisito previo para la comisión de delitos Según Warr (1996), si se analiza desde la perspectiva del grupo y no del individuo, los grupos favorecen una delincuencia juvenil especializada, los jóvenes de cada grupo no cometen sino determinados tipos de delitos bien definidos y reconocidos por éste. En consecuencia, se constata pues que un joven pertenenciendo a un solo grupo de jóvenes tendrá tendencia a adoptar una conducta delincuente especializada. El hecho de frecuentar siempre a los mismos compañeros o amigos significa que el joven es confrontado a los mismos tipos de delitos y aprende a cometerlos. La versatilidad de la delincuencia se explica a su vez por el hecho de pertenecer a varios grupo delincuentes diferentes. Estas personas aprenden a cometer lo delitos particulares propios de cada grupo frecuentado, dándole la oportunidad de tener una actividad delictiva muy cambiante. Volviendo a los cambios en el estilo de vida de un joven, que puede ser consecuencia de un cambio del grupo de amigos a causa de una dispu- ta dentro del primer grupo, el aburrimiento o la simple disolución de este último. Esto puede explicar los cambios en los tipos de delitos cometidos o la cesación de la participación en la delincuencia delito, si el nuevo grupo ocupa su tiempo libre en actividades legales.

La segunda corriente de ideas que se estudió en esta investigación es que el hecho de pertenecer a un grupo engendra una delincuencia versátil. Esto brinda experiencias y un aprendizaje variado de la delincuencia. Los miembros tendrían tendencia a tener una delincuencia más versátil debido a las diferentes influencias que ellos encuentran en el seno del grupo, donde las relaciones son volátiles y las afiliaciones transitorias (Reiss, 1986), estas interacciones proporcionan un amplio espacio para el desarrollo de influencias mutuas muy variadas. Por el contrario, los indididuos que tienen sólo unos pocos amigos, sin que ellos constituyan un grupo, tienen una delincuencia más especializado, debido al hecho de que sufren menos influencias diferentes y tienen un aprendizaje social de la delincuencia limitada a los pocos amigos que el frecuenta (Klein y Crawford, 1967 citado por Warr, 1996; Reiss 1986).

\section{La teoría de la elección racional}

La teoría de la elección racional se basa en un modelo económico extraido de las ciencias sociales. Los defensores de este enfoque se centran en el apriori de que la comisión de un delito sirve principalmente a necesidades materiales. Este modelo se basa en la idea de que toda acción es fundamentalmente racional, el individuo calcula los costos y beneficios probables de cada una de sus acciones antes de ejecutarla (Scott, 2000). Más específicamente a nivel criminológico, los investigadores tratan de explicar el proceso cognitivo que conduce al delincuente a pasar al acto.

Estos formulan dos ideas principales que describen los momentos claves, donde una elección racional es operada por el individuo. La primera perspectiva llamada "participación criminal" es la detallada en este estudio. La otra perspectiva llamada "evento criminal", describe el cálculo mental que lleva a decidir al delincuente a cometer un delito si los beneficios que aporta, son mayores que los costos.

La implicación criminal -en inglés «criminal involvement»se fundamenta en las creencias, valores y códigos morales del individuo (Cornish y Clarke, 1985). Un individuo con creencias, moral o valores que tienden a aceptar la comisión delitos para satisfacer necesidades personales, tendrá pues tendencia a implicarse fácilmente en conductas delictivas.

En esta investigación se puede deducir que una personaalores tolerantes a los delitos de mayor o menor gravedad tendrá tendencia a implicarse en una delincuencia versá- 
til. Si a esto se suman las necesidades del delincuente que puden ser para algunos muy grandes (necesidad de dinero, satisfacción sexual, el reconocimiento de la gente, búsqueda de sensaciones, la codicia) el individuo puede ser implusado a cometer delitos de naturaleza muy diversa.

Tengase en cuenta también que las experiencias positivas durante las cuales los hechos criminales refuerzan al joven en la creencia de que fácil obtener satisfacción de sus propios deseos a través de la comisión de delitos. Estas experiencias vividas de más en más positivamente son a veces susceptibles de cambiar los valores, creencias y códigos morales de una persona por un mecanismo psicológico de racionalización. En efecto, el individuo va a cambiar sus cogniciones de tal forma que tendrá pensamientos que justifican su comportamiento. Por lo tanto, él participará en una actividad delictiva cada vez más extendida y diversa, sin que esto le plantee demasiados problemas de conciencia, teniendo asi un poderoso mecanismo de racionalización que lo protege de todo cuestionamiento moral.

En resumen, el delincuente versátil es un individuo que tiene una propensión a cometer delitos y a medida que desarrolla su actividad criminal esta en capacidad de cambiar sus ideas básicas para justificar sus acciones.

Valores, creencias y códigos morales son el resultado de diferentes factores. Cornish y Clarke (1985) señalan tres principales:

1) Factores psicológicos: el temperamento, la inteligencia y el estilo cognitivo

2) Los factores educativos: cuidados educativos, dinámica familiar y criminalidad parental

3) Los factores sociodemográficos: sexo, clase social, nivel de educación y medio ambiente.

Acerca de los delincuentes especializados en ciertos tipos de delitos específicos, los investigadores (Cornish y Clarke, 1985) tratan de explicar sus actividades según diferentes niveles de funcionamientos.

En primer lugar, en todo proceso educativo, las creencias, valores y los códigos morales se construyen en los delincuentes especializados sobre la base de factores psicológicas, educativos y sociodemográficos menos caóticos y más prosociales que en los delincuentes versátiles.

El individuo con una actividad delictiva especializada desarrolla pues valores morales más "convencionales" y se implicará con más reticencia en comportamientos delictivos. La gama de delitos que cometerá será también más limitada de lo que puede ser en los delincuentes versátiles. Los actos son a menudo sin mucha violencia, de poca gravedad y se los comete principalmente para satisface necesidades financieras y por el deseo de obtener un estatus privilegiado entre sus pares que perciben la transgresión de la prohibición como algo "heroico". Asi pues, estos delincuentes no amplían su actividad delictiva como los delincuentes versátiles. Esto se explica no sólo por sus valores morales que no abogan por tales comportamiento, que llegan incluso a considerar algunos inaceptables, sino también a nivel de experiencias criminales vividas. De hecho, las primeras experiencias delincuentes pueden a veces no haber sido vividas positivamente. Esto por diversas razones, como por ejemplo haber sido atrapado en flagrante delito y haber sido castigado severamente o por el costo moral que les ha valido la infracción, o los remordimientos habiéndolo de alguna manera "traumatizado". Asi un individuo versátil, teniendo valores positivos hacia la delincuencia puede ser también frenado por las experiencias criminales vividas. Los delincuentes especializados se limitan a cometer exclusivamente ciertos delitos que ellos consideran aceptables. Entre ellos, los delitos por los que no han sido aprendidos y tienen menos riesgo de ser castigados. Esto es consistente con la idea del evento criminal («Criminal involvement»), lo que significa que el delincuente, frente a una oportunidad de cometer un delito, no selecciona que esos susceptibles de satisfacer un maximun sus necesidades, y esto por un riesgo mínimo.

En fin la ultima faceta de la teoría de la elección racional que no es objeto de esta investigación, explica el desistimiento en la implicación criminal y el evento criminal. En resumen, las personas que no se implican en comportamientos delictivos son aquella que tienen creencias, valores y códigos morales que consideran los comportamientos delictivos como simplemente inaceptables y contrarios a su percepción del mundo.

\section{Metodología}

\section{Población}

La ISRD-2 es una encuesta internacional de delincuencia juvenil autoinformada. En Suiza se realizó con una muestra nacional de más de 3.000 jóvenes estudiantes de los grados $7^{\circ}, 8^{\circ}, y^{\circ}$, de edades comprendidas entre los 13 y 16 años, esta encuesta fue realizada por el Instituto de Criminología y Derecho penal de la Universidad de Lausanne (Killias, Aebi, Herrmann, Dilitz y Lucía, 2009).

La muestra Suiza esta compuesta por 3648 jóvenes de 70 escuelas de 20 cantones, lo que representan 2.549 cuestionarios en alemán, 806 en francés y 293 en italiano. La mayoría de los estudiantes tenían edades comprendidas entre los 13 y los 16 años, pero algunos tenian 12 años $(2,5 \%)$ y otros $17(1,8 \%)$ (Killias et al., 2009). 
Tengase en cuenta que no se trata de una población de delincuente propiamente dicho, sino de una población de jovenes ordinarios, comúnes y corrientes, que están todavía en la escuela.

En el contexto específico de este estudio, se ha depurado la base de datos para tener en cuenta en el análisis solo individuos que han cometido al menos un delito en los últimos doce meses o consumido drogas durante las últimas cuatro semanas antes de la aplicación de la encuesta. La prevalencia de vida no se considera aquí en aras de estudiar sólo el período de vida de los jóvenes en constante evolución a esta edad. La muestra sobre la que se trabajó tiene sinembargo 829 individuos.

\section{Las hipótesis}

A fin de determinar si las diversas teorías presentadas se aplicaban a la muestra, se extrajeron de cada una de ellas, hipótesis que permitian probarlas estadísticamente.

- La primera hipótesis se relaciona con las teorías del desarrollo que indican una diferencia en cuanto a la frecuencia, la gravedad y la edad de iniciación en la actividad criminal. De acuerdo con estas teorías, en este estudio se esperaba que los delincuentes versátiles cometieran sus primeros delitos a una edad más temprana, con una frecuencia más elevada y con más violencia que los delincuentes especializados

- La segunda hipótesis se basa en la teoría del autocontrol que trata de la personalidad misma del individuo. Se esperaba que los delincuentes versátiles presentaran un nivel de autocontrol más bajo que los jovenes que cometen un tipo determinado de delitos es decir que tienen una delincuencia especializada.

- La tercera hipótesis relacionada con la teoría de las actividades rutinarias descrita por el enfoque o las teorías situacionales. En concreto, en este trabajo el interes se ha focalizado en las frecuentaciones que pudieran tener los jóvenes de la muestra. Se quizo probar si efectivament el hecho de pertenecer a un grupo engendra una delincuencia versátil mientras que la delincuencia especializada es el resultado del hecho que el joven no frecuenta sino a algunos amigos sin que estos llegen a constituir un grupo.

- Por último, la cuarta hipótesis se refiere a la teoría de la elección racional que sugiere que la diferencia entre los delincuentes especializados y los delincuentes versátiles radica en la tolerancia que tienen los individuos hacia la violencia. Se supone entonces que los individuos que tienen una delincuencia versátil aceptan más los comportamientos violentos mientras que los delincuentes especializados presentan una tolerancia más baja hacia éstos.

\section{Diversidad}

La pregunta principal de este estudio gira en torno a la versatilidad y la especialización de la delincuencia. La principal literatura sobre este tema, por otro lado relativamente específica, utilice el «Forward Specialization Coefficient» (Paternoster, Brame, Piquero, Mazerolle y Dean, 1998). Se trata de matrices matemáticas que tienen en cuenta tanto el tiempo como el tipo de delitos cometidos. De hecho, la mayoría de los estudios se basan en los datos de la policía. Los investigadores (Farrington, Snyder y Finnegan, 1988; Kempf, 1987, Blumstein et al, 1986) no hacen sino constatar si de un arresto a otro los individuo han o no cometido los mismos delitos.

Se toma como variable dependiente la tasa de diversidad. Esta variable mide la amplitud de la gama de delitos cometidos por cada individuo. Según Aebi (2006): "El índice de diversidad es interesante para el estudio de la delincuencia en la medida en que la gama de delitos cometidos permite evaluar el grado de implicación en el conjunto de la delincuencia. A diferencia del cálculo de la incidencia, el de la diversidad no está influenciado por ciertas respuestas poco fiables sobre el número de veces en que un delito ha sido cometido." En este estudio, la diversidad se midió sobre la base de la prevalencia de los últimos doce meses de 15 infracciones presente en la ISRD-2. Se trata del hurto, la agresión, el vandalismo, el raponazo, el hurto a almacenes, el porte de arma, el robo de bicicleta, ciclomotor o scooter, el robo de moto o de automovil, el robo dentro de un automovil, la riña en grupo, la piratería informática, el robo de apartamento, la venta de drogas, el consumo de LSD, cocaína o heroína (todo esto agrupado en una pregunta) $y$, finalmente, el consumo de éxtasis o speed (anfetamina).

Después de la depuración de la base de datos, se obtiene una muestra de 829 individuos que habian cometido durante los últimos 12 meses, (contados a la fecha de la encuesta) uno de los 15 delitos seleccionados para el análisis.

Por último, se operacionalizó la escala de la diversidad de la siguiente manera: los delincuentes especializados son aquellos que, durante los últimos doce meses, no han cometido que uno o dos delitos diferentes. Ellos represen$\tan 629$ individuos y constituyen el 82,3\% de la muestra. Los delincuentes versátiles son los que han cometido al menos tres tipos de delitos. Se cuentan 147 y representan el $17,7 \%$ de la muestra de 829 individuos.

\section{Las variables independientes}

Para probar las cuatro hipótesis, se seleccionaron seis variables independientes. 
Tabla 1. Frecuencia de las variables independientes

\begin{tabular}{|l|c|c|c|c|c|c|c|c|}
\hline \multicolumn{2}{|c|}{ Edad de inicio } & incidencia & gravedad & $\begin{array}{c}\text { Nivel de } \\
\text { autocontrol }\end{array}$ & \multicolumn{2}{c|}{ Grupo de pares } & \multicolumn{2}{c|}{$\begin{array}{c}\text { Tolerancia hacia } \\
\text { la violencia }\end{array}$} \\
\hline Precoz & $\begin{array}{c}30.2 \% \\
(\mathrm{~N}=247)\end{array}$ & $\begin{array}{c}56.5 \% \\
(\mathrm{~N}=468)\end{array}$ & $\begin{array}{c}59.5 \% \\
(\mathrm{~N}=472)\end{array}$ & $\begin{array}{c}54.1 \% \\
(\mathrm{~N}=444)\end{array}$ & Individualista & $\begin{array}{c}24.3 \% \\
(\mathrm{~N}=201)\end{array}$ & Debil & $\begin{array}{c}18.3 \% \\
(\mathrm{~N}=149)\end{array}$ \\
\hline Tardio & $\begin{array}{c}69.8 \% \\
(\mathrm{~N}=573)\end{array}$ & $\begin{array}{c}26.2 \% \\
(\mathrm{~N}=217)\end{array}$ & $\begin{array}{c}40.5 \% \\
(\mathrm{~N}=320)\end{array}$ & $\begin{array}{c}45.9 \% \\
(\mathrm{~N}=376)\end{array}$ & $\begin{array}{c}\text { Grupo no } \\
\text { delincuente }\end{array}$ & $\begin{array}{c}28.4 \% \\
(\mathrm{~N}=235)\end{array}$ & Media & $\begin{array}{c}58.9 \% \\
(\mathrm{~N}=481)\end{array}$ \\
\hline & $\begin{array}{c}17.3 \% \\
(\mathrm{~N}=143)\end{array}$ & & & $\begin{array}{c}\text { Grupo } \\
\text { delincuente }\end{array}$ & $\begin{array}{c}47.4 \% \\
(\mathrm{~N}=393)\end{array}$ & Fuerte & $\begin{array}{c}22.8 \% \\
(\mathrm{~N}=186)\end{array}$ \\
\hline $\begin{array}{l}\text { Valores } \\
\text { faltantes }\end{array}$ & 9 & 1 & 37 & 9 & & 0 & & 13 \\
\hline Total & 829 & 829 & 829 & 829 & & 829 & & 829 \\
\hline
\end{tabular}

\subsection{La edad de inicio}

La variable edad de inicio, en inglés ««onset age»» traduce la edad promedio en la que un joven comete por primera vez delitos. Esta variable se presentó en forma dicotómica en el cuestionario. Siendo de una parte los delincuentes precoces que van hasta un promedio de 12 años de edad. Ellos representan $30.2 \%$ de la muestra, o sea 247 delincuentes. Por otro lado los delincuentes tardíos, habiendo cometidos sus delitos en una edad superior a 12 años. Se han contado 573 o sea $69,8 \%$ de la muestra. Se ha elegido el límite de 12 años porque esto corresponde a la pubertad. Se trata del momento en que los principales cambios biológicos y sociales ocurren. Los investigadores (Piquero et al., 1999) también consideran igualmente esta edad como un buen límite para diferenciar las dos subpoblaciones.

\subsection{La incidencia}

La variable de la incidencia mide la frecuencia de la comisión de los delitos durante los doce meses anteriores a la administración de la encuesta. En otras palabras, se mide la intensidad de la actividad criminal de cada individuo de la muestra. Esta variable se codificó en tres categorías: la primera incluye a los individuos habiendo cometido entre 1 y 3 actos delictivos, que representan un total de 468 individuos, o sea $56.5 \%$ de la muestra. El segundo es para aquellos que cometieron entre 4 y 10 actos delictivos es decir 217 personas o sea $26.2 \%$. Por último, la tercera categoría incluye a los que han cometido entre 11 y 100 delitos en los últimos doce meses, (o anteriores a la administración de la encuesta) se contaron 143, es decir el 17.3\%-

\subsection{La gravedad}

La cuestión de la gravedad de los delitos cometidos por los jóvenes es un elemento clave de este estudio, esto., debido al hecho de que la mayoría de los estudios sobre la especialización y la diversidad de la delincuencia muestra una fuerte dependencia entre estas dos nociones La operacionalización de esta variable se efectuó sobre la base de la prevalencia de los últimos doce meses de los cuatro delitos considerados como los más violentos entre los quince tenidos en cuenta en el análisis. Se trata de la agresión, el hurto, las riñas en grupo y el raponazo.. Los individuos habiendo cometido uno de estos cuatro delitos durante los doce meses anteriores a la encuesta se agruparon en la categoría de «delincuentes violentos», los otros en la de los «delincuentes no violentos». La primera con 472 individuos mientras que la segunda tiene 320 respectivamente $59,5 \%$ y $40,5 \%$ de la muestra (Tabla1).

\subsection{El nivel de autocontrol}

La variable de autocontrol tal como fue medida en la encuesta agrupa varias preguntas relacionadas con la impulsividad, el egocentrismo y la toma de riesgos que refleja el temperamento de cada individuo. Las nueve preguntas formuladas en el ISRD-2 cosntituyen una escala de autocontrol dividida en dos categorías. Por un lado el «alto nivel de autocontrol» que representa 444 jóvenes $(54,1 \%)$ con una puntuación en la parte inferior de la escala, por otra parte, el «bajo nivel de autocontrol» con 376 individuos $(45,9 \%)$ habiendo obtenido una alta puntuación.

\subsection{El grupo de pares}

La variable referente a a grupo de pares que frecuenta cada joven se formó sobre la base de cuatro preguntas del ISRD-2 permitiendo de establecer el entorno social en el que este evoluciona. Las preguntas son las siguientes: la pregunta 27: «Algunas personas tienen un determinado grupo de amigos con los que pasan el tiempo, con los que realizan actividades o van de un lado a otro. ¿Tienes tú tal grupo de amigos? «Pregunta 31: « ¿hacer cosas prohibidas (ilegales) es aceptado o tolerado en tu grupo de amigos?". 
Pregunta 32: «iEs que en tu grupo, hay personas que hacen cosas prohibidas (ilegales) juntas?". Finalmente la pregunta 33: «¿Crees que tu grupo de amigos es una pandilla?

A raíz de los resultados obtenidos, la variable «grupo de pares» se dividió en tres categorías: los «individualistas» son jóvenes que respondieron no a la pregunta 27, ellos constituyen el 24,3\% de la muestra, o sea 201 individuos. La categoría "grupos de no delincuentes" agrupa los jóvenes no comentiendo actos delictivos, incluso si estos son aceptados en el seno de su grupo. Se contaron en esta categoria 235 o sea $28,4 \%$ de la muestra. Por último, la categoría «grupos de delincuentes» incluye a los jóvenes que cometen delitos. Representan el 47,4\% de la muestra para un total de 393 delincuentes.

\subsection{La tolerancia de la violencia}

Esta variable mide la tolerancia del individuo hacia la violencia. No hay en la encuesta ISRD-2 pregunta acerca de la tolerancia general hacia la delincuencia. A pesar de esto, parece correcto pensar que una evaluación favorable hacia la tolerancia engloba también valores positivos hacia los otros tipos de delitos más triviales. Así se podría generalizar la tolerancia hacia la violencia como una aceptación moral de la delincuencia en general.

Se ha construido esta variable sobre la base de cinco preguntas. La pregunta 38.1 «iun poco de violencia es parte del juego» la pregunta 38.2: «Si queremos ser respetados, se debe utilizar la fuerza» la pregunta 38.3: «Si alguien me ataca, yo reacciono golpeando», la pregunta 38.4 «sin violencia, todo sería mucho más aburrido ", y finalmente la pregunta 38.5 «Es completamente normal que uno quiera mostrar que se es un hombre peleandose con los demás». Seguidamente se han agrupado las diferentes respuestas para obtener una única variable que se ha dividido en tres categorías. La primera, llamada «baja tolerancia», incluye 149 individuos, o sea el 18,3\% de la muestra tiene una puntuación de entre 0 y 9 (Tabla 1). La segunda se refiere a los jóvenes con una «tolerancia media» a la violencia. La puntuación de este último es de entre 10 y 99 (Tabla 1). Ellos representan 481 personas, o sea el $58,9 \%$ de la muestra. Por último la categoría «alta tolerancia» incluye a las personas que obtuvieron una puntuación entre 100 y 122, que cuenta con 186 adolescentes o sea el 22,8\% de la muestra (Tabla 1).

\section{Resultados}

\subsection{Perfiles de los delincuentes y la edad de iniciación en la delincuencia}

La edad de inicio en la delincuencia, es según las teorías del desarrollo (Gottfredson, 2005; Moffitt, 1993, 1997, Tremblay, 2000; Farrington 1986, Blumstein et al. 1986. Piquero et al. 1999; Monahan y Piquero, 2009), uno de los principales predictores de la delincuencia futura de un individuo. En efecto, se sugiere que cuanto más una persona se implica en una actividad criminal a una edad temprana, tien más probabilidad de tener una delincuencia versátil y violenta en la adolescencia. Por el contrario, las personas que cometen su primer delito a una edad más tardia tienen una delincuencia futura relativamente especializada. El límite establecido por los investigadores para discernir si se trata de una delincuente precoz o tardío se situa hacia los 12 años.

Para probar estos postulados teóricos en este estudio, se comparan los dos grupos de interés a fin de determinar en qué medida se diferencian en función de la edad de inicio en la delincuencia.

Tabla 2. Test de Anova de la edad de inicio (control por sexo)

\begin{tabular}{|c|c|c|c|c|c|}
\hline & Promedio & Mínimo & Máximo & $\mathbf{F}$ & Significación \\
\hline Niñas & & & & & \\
\hline Del. especializados $(\mathrm{N}=264)^{1}$ & 13.09 & 6 & 16 & \multirow{2}{*}{0.001} & \multirow{2}{*}{0.970} \\
\hline Del. versátiles $(\mathrm{N}=33) 1$ & 13.08 & 10 & 15 & & \\
\hline \multicolumn{6}{|l|}{ Niños } \\
\hline Del. especializados $(\mathrm{N}=409)^{1}$ & 12.78 & 7 & 16 & \multirow{2}{*}{1.162} & \multirow{2}{*}{0.282} \\
\hline Del. versátiles $(\mathrm{N}=113)^{1}$ & 12.59 & 4 & 15 & & \\
\hline
\end{tabular}

${ }^{1}$ El número total de delincuentes se eleva a 819 debido a la utilización de los valores redondeados para el análisis, la base de datos estanto ponderada. 
La prueba de ANOVA (Tabla 2) muestra que no hay diferencia significativa en términos de promedio de edad de iniciación en la delincuencia entre los delincuentes versátiles y especializados, independientemente del sexo del individuo. Los resultados que se han obtenido difieren ligeramente de los obtenidos por los investigadores estadounidenses. Tenga en cuenta, sin embargo, un resultado que es contrario a las expectativas que se tenían en este estudio: se trata de las edades de iniciación mínima de los jóvenes delincuentes de sexo femenino. Se percibe que las delincuentes versátiles tienen una edad de iniciación minima de 10 años, mientras que los especializadas tienen una edad de iniciación minima de 6 años. Esta edad de iniciación minima situado en los 6 años en las niñas es igualmente inferior a la edad de inciciación minima en la delincuencia de los delincuentes esécializados de xeso masculino. De hecho, se esperaba a que las niñas con una delincuencia especializada presenten una edad de iniciación mínima en la delincuencia superior a todos los otros grupos.

Los resultados presentados en la Tabla 3. no uestranr ninguna diferencia significativa entre los delincuentes especializados y versátiles en cuanto a la edad de iniciación en la delincuencia. Se observa que el 30,4\% de los delincuentes especializados contra $28,8 \%$ delincuentes versátiles inició sus actividades delictivas a una edad temprana. Aunque se desprende de estos resultados que hay más delinceuntes precoces en el grupo de los especializados que en los delincuentes versátil, esta diferencia de proporciones no fue estadísticamente significativa $(p>0,10)$. Parece que, independientemente de la edad a la que una persona se inicia en la actividad delictiva, tampoco tiene ninguna influencia en el tipo de delitos adoptado en la adolescencia. Recuerdese que la mayoría de autores prue- ban sus observaciones o hypotesis sobre delincuentes reincidentes o personas conocidas por las autoridades. La ISRD-2 sondea individuos ordinarios. Cualquier diferencia entre los dos grupos de delincuentes podría aparecer en una población ordinaria de más edad

\subsection{Perfiles de los delincuentes y el impacto de la actividad criminal}

Moffitt (1993, 1997), Tremblay (2000), Farrington (1986), Blumstein et al. (1986), Piquero et al. (1999), Monahan y Piquero (2009) sugieren que los delincuentes versátiles cometen delitos con una frecuencia superior al delincuente especializado.

La variable «incidencia» que permite probar estas observaciones tiene una fuerte dependencia con la variable dependiente «diversidad». Sin embargo, se juzgan satisfactorios los resultados satisfactorios, dada la población estudiada. En efecto, recuérdese que se trata de jóvenes que van todavía a la escuela y no conocidos de las autoridades. A pesar de una metodología cuestionable, las diferencias observadas no se pueden pasar por alto.

La Tabla 4 muestra que si se compara los promedios del numero de delitos cometidos por los dos grupos de delincuentes del estudio, la diferencia entre ellos es significativa. Los delincuentes especializados cometen en promedio 5 en el espacio de doce meses, mientras que los delincuentes versátiles, cometen un promedio de 29 en el mismo período. En efecto, existe una gran diferencia en cuanto a la frecuencia de comisión de delitos entre los dos tipos de delincuentes.

Tabla 3. Edad de iniciación y diversidad

\begin{tabular}{|l|c|c|c|}
\hline & Delincuentes precoces & Delincuentes tardíos & \\
\hline Dél. especializada $(\mathrm{N}=675)$ & $30.4 \%(\mathrm{n}=205)$ & $69.6 \%(\mathrm{n}=470)$ & $100 \%$ \\
\hline Dél. versatiles $(\mathrm{N}=146)$ & $28.8 \%(\mathrm{n}=42)$ & $71.2 \%(\mathrm{n}=104)$ & $100 \%$ \\
\hline Test de proporcion & \multicolumn{2}{|c|}{$\mathrm{Z}=0.39$} \\
\hline Nivel de signification & $\mathrm{p}>0.10$ & \\
\hline
\end{tabular}

Tabla 4. Test Anova de la incidencia

\begin{tabular}{|l|c|c|c|c|c|}
\hline & Promedio & Mínimo & Máximo & F & Significación \\
\hline Dél. especializados (N=683) & 5.1 & 1 & 70 & \multirow{2}{*}{294.452} & 0.00 \\
\hline Dél. versatiles (N=147) & 28.6 & 3 & 100 & & \\
\hline
\end{tabular}


Tabla 4.1. Test Anova de la incidencia (según el sexe)

\begin{tabular}{|c|c|c|c|c|c|}
\hline & Promedio & Mínimo & Máximo & F & Significación \\
\hline \multicolumn{6}{|l|}{ Femenino } \\
\hline Dél. especializadas (N=267) & 4.26 & 1 & 60 & \multirow[b]{2}{*}{51.252} & \multirow[b]{2}{*}{0.000} \\
\hline Dél. versatiles $(\mathrm{N}=33)$ & 18.76 & 3 & 100 & & \\
\hline \multicolumn{6}{|l|}{ Masculino } \\
\hline Dél. especilizados (N=415) & 5.66 & 1 & 70 & \multirow[b]{2}{*}{208.831} & \multirow[b]{2}{*}{0.000} \\
\hline Dél. versatiles $(\mathrm{N}=113)$ & 31.15 & 3 & 100 & & \\
\hline
\end{tabular}

Resultados interesantes aparecen cuando se controlan las diferencias según el género, la prueba de ANOVA (Tabla 4.1). Muestra que el hecho de ser un varon tiene más influencia en la frecuencia de comisión de delitos que si se es una niña. Las delincuentes especializadas cometen en promedio 4 delitos en doce meses, mientras que los delincuentes especializados comenten en promedio 6. En los versátiles, sus diferencias de promedios son aún mayores, las niñas cometen un promedio de 19 al año, mientras que los chicos cometen 31 en el mismo período.

Tabla 4.2. Incidencia y diversidad

\begin{tabular}{|l|c|c|c|c|}
\hline & $\mathbf{1}$ à $\mathbf{3}$ delitod $^{1}$ & $\mathbf{4}$ à $\mathbf{1 0}$ delitos & $\mathbf{1 1}$ à $\mathbf{1 0 0}$ delitos & \\
\hline Niñas & & & & \\
\hline Dél. especializadas (N=267) & $73.0 \%(\mathrm{n}=195)$ & $19.9 \%(\mathrm{n}=53)$ & $7.1 \%(\mathrm{n}=19)$ & $100 \%$ \\
\hline Dél. versatiles (N=32) & $3.1 \%(\mathrm{n}=1)$ & $65.6 \%(\mathrm{n}=21)$ & $31.1 \%(\mathrm{n}=10)$ & $100 \%$ \\
\hline Test de proporciones & $\mathrm{Z}=17.07$ & $\mathrm{Z}=5.23$ & $\mathrm{Z}=2.89$ & \\
\hline Nivel de significación & $\mathrm{p}<=0.001$ & $\mathrm{p}<=0.001$ & $0.001<\mathrm{p}<=0.01$ & \\
\hline Varones & & & & \\
\hline Dél. especializados (N=414) & $64.5 \%(\mathrm{n}=267)$ & $24.2 \%(\mathrm{n}=100)$ & $11.4 \%(\mathrm{n}=47)$ & $100 \%$ \\
\hline Dél. versatiles (N=113) & $1.8 \%(\mathrm{n}=2)$ & $38.9 \%(\mathrm{n}=44)$ & $59.3 \%(\mathrm{n}=67)$ & $100 \%$ \\
\hline Test de proporcion & $\mathrm{Z}=23.54$ & $\mathrm{Z}=2.91$ & $\mathrm{Z}=9.82$ & \\
\hline Nivel de significación & $\mathrm{p}<=0.001$ & $0.001<\mathrm{p}<=0.01$ & $\mathrm{p}<=0.001$ & \\
\hline
\end{tabular}

${ }^{1}$ Entre los delincuentes especializados que cometieron entre 1 y 3 delitos sólo el 16,8\% han cometido tres delitos. Por lo tanto, no se va a construir un grupo especializado de delincuentes fuertemente involucrados en el crimen, esto no es útil.

En la Tabla 4.2 se encuentran diferencias en el mismo nivel de análisis entre las niñas y los niños. Es decir, hay mucho más especializados delincuentes que cometen delitos entre el 1 y 3 durante los últimos doce meses. Delincuentes especializados son $73,0 \%$ contra $3,1 \%$ delincuentes versátiles de haber cometido crímenes de 1-3 años anteriores a la encuesta ( $p<=0,001)$. Entre los varones, la diferencia está en el mismo nivel de importancia, especializada contra $64,5 \% 1,8 \%$ versátil que ha cometido el mismo número de delitos en el mismo período de tiempo.
Las diferencias se invierten en las dos categorías restantes de la variable «incidencia». A saber que los delincuentes versátiles son más numeroso a cometer entre 4 y 10 delitos y entre 11 y 100 delitos durante el período estudiado. Para las niñas, se cuenta $65,5 \%$ de las delincuentes versátiles frente a 19,9\% de las delincuentes especializadas habiendo cometido entre 4 y 10 durante los últimos doce meses ( $p<=0,001$ ). Los varones fueron $38,9 \%$ y $24,2 \%$ respectivamente $(0,001<\mathrm{p}<=0,01)$. 
En cuanto a la última categoría de la variable «incidencia» reagrupando los individuos que cometieron de 11 a 100 delitos el año anterior a la administración de la encuesta, las niñas con delincuencia versátil cosntituyen el 31,3\% en esta categoría frente a sólo el 7,1\% de las especializadas $(0,001<p<=0,01)$. Los varones con una delincuencia versátil son $59,3 \%$ contra $11,4 \%$ durante los últimos doce meses $(\mathrm{p}<=0,001)$.

La Tabla 3.2 muestra la influencia del género, se observa claramente que los niños, sin importar su tipo de delincuencia, cometen en general delitos con una mayor frecuencia que las niñas. En las delintes especializadas, se nota que las niñas se observamos que las niñas son más numerosas entre las que cometieron entre 1 a 3 delitos en 12 meses. Mientras que los varones se encuentran en mayor proporción en las dos últimas categorías de la variable, es decir, hay una actividad delictiva más frecuente bien que especializa en la comisión de uno a dos tipos de delitos.

En los delincuentes versátiles, las niñas, son más propensos en las dos primeras categorías de la variable (entre 1 y 10 delitos), mientras que en la categoría 11 a-100 delitos hay dos veces chicos dos veces más varones que niñas haciendo parte de ésta.

\subsection{Perfiles delincuentes y gravedad de la delincuencia}

Moffitt (1993, 1997), Tremblay (2000) y Guerette et al. (2005) plantean que los delincuentes versátiles tienen una actividad delictiva más violenta que los delincuentes, especializados, se ha sometido a test esta hipótesis mediante la comparación de los dos grupos de interés para ver si difieren en cuanto a la actividad criminal violenta.
Se ve en la Tabla 5 que tantos en los niños como en las niñas, los jóvenes con delincuencia versátil son más numerosos a haber cometido uno de los cuatro crímenes violentos que se ha seleccionado. De hecho, ellos son 63,3\% en el grupo de las mujeres y $80,0 \%$ en los varones, contra solamente $22,9 \%$ y $39,9 \%$, respectivamente, en los delincuentes especializados $(\mathrm{p}<=0,001)$.

Sin embargo, se observa que el hecho de ser un varón crea una delincuencia aún más violenta que el hecho de una niña, independientemente del tipo de delincuencia adoptada.

En los delincuentes especializados, los varones (39,9\%) son casi dos veces más numerosos que las niñas $(22,9 \%)$ habiedno cometido uno de los cuatro delitos violentos durante los últimos doce meses. Los delincuentes versátiles de sexo masculino $(80,0 \%)$ son igualmente más numerosos que las niñas $(63,3 \%)$ en tener una actividad criminal violenta (Tabla 5).

\subsection{Perfiles delincuentes y Teoría del autocontrol}

El control interno que todo el mundo sería capaz de autoadministrarse tendría, según los defensores de la teoría del autocontrol (Gottfredson y Hirschi, 1990; McGloin et al, 2007;. Mason y Windle, 2002; Mazerolle et al. , 2000), una gran influencia en el tipo de actividad criminal adoptada por los delincuentes. Para probar esta afirmación, se compararon los dos grupos de delincuentes para ver si difieren en términos del nivel de auto-control.

Tabla 5. Gravedad y diversidad

\begin{tabular}{|c|c|c|c|}
\hline & Délincuentes no-violentos & Delincuentes violentos & \\
\hline \multicolumn{4}{|l|}{ Niñas } \\
\hline Dél. espécializadas (N=262) & $77.1 \%(n=202)$ & $22.9 \%(n=60)$ & $100 \%$ \\
\hline Dél. versatiles $(\mathrm{N}=30)$ & $36.7 \%(\mathrm{n}=11)$ & $63.3 \%(n=19)$ & $100 \%$ \\
\hline Test de proporción & \multicolumn{2}{|c|}{$Z=4.40$} & \\
\hline Nivel de significación & \multicolumn{2}{|c|}{$\mathrm{p}<=0.001$} & \\
\hline \multicolumn{4}{|l|}{ Varones } \\
\hline Dél. espécializadas (N=393) & $60.1 \%(n=236)$ & $39.9 \%(n=157)$ & $100 \%$ \\
\hline Dél. versatiles ( $\mathrm{N}=105)$ & $20.0 \%(\mathrm{n}=21)$ & $80.0 \%(\mathrm{n}=84)$ & $100 \%$ \\
\hline Test de proporción & \multicolumn{2}{|c|}{$Z=8.68$} & \\
\hline Nivel de significación & \multicolumn{2}{|c|}{$\mathrm{p}<=0.001$} & \\
\hline
\end{tabular}

${ }^{1}$ Délincuentes habiendo cometido al menos un de los cuatro delitos siguientes: asalto, robo, riñas o pleas en grupo, robo con violencia. 
Tabla 6. Nivel del autocontrol y diversad

\begin{tabular}{|l|c|c|c|}
\hline & Bajo nivel de autocontrol & Alto nivel de autocontrol & \\
\hline Filles & & & \\
\hline Dél. especializadas (N=264) & $39.0 \%(\mathrm{n}=103)$ & $61.0 \%(\mathrm{n}=161)$ & $100 \%$ \\
\hline Dél. versatiles (N=33) & $66.7 \%(\mathrm{n}=22)$ & $33.3 \%(\mathrm{n}=11)$ & $100 \%$ \\
\hline Test de proporcion & \multicolumn{2}{|c|}{$\mathrm{Z}=3.17$} \\
\hline Nivel de significación & \multicolumn{2}{|c|}{$0.001<\mathrm{p}<=0.01$} & \\
\hline Varones & $40.1 \%(\mathrm{n}=164)$ & $59.9 \%(\mathrm{n}=245)$ & $100 \%$ \\
\hline Dél. especializados (N=409) & $77.7 \%(\mathrm{n}=87)$ & $22.3 \%(\mathrm{n}=25)$ & $100 \%$ \\
\hline Dél. versatiles (N=112) & \multicolumn{2}{|c|}{$\mathrm{Z}=8.14$} \\
\hline Test de proporción & $\mathrm{p}<=0.001$ \\
\hline Nivel de significación & \multicolumn{2}{|c|}{} \\
\hline
\end{tabular}

La Tabla 6 muestra a nivel de la población femenina, que las delincuentes versátiles $(66,7 \%)$ son más numerosas las que presentan un bajo nivel de autocontrol que las delincuentes especializadas que cuentan $39,0 \%(0.001<\mathrm{P}<=$ $0,01)$.

Entre los varones, los versátiles son $77,7 \%$ a tener un nivel bajo de autocontrol contra un $40,1 \%$ en los delincuentes especializados $(\mathrm{p}<=0,001)$.

Se constata pues que tantos los chicoscomo las chicas son los jóvenes con una delincuencia versátil están más relacionados con el bajo nivel de auto-control.

\subsection{Perfiles delincuentes y las teorías situacionales}

Las teorías situacionales abordan una multitud de elementos que pueden llevar a una persona a la delincuen- cia. Entre ellos, este estudio se interesó en los hipótesis propuestas por Warr (1996) y Reiss (1986) y trató de conoceren que medida el universo social en el cual se desenvuelve el jóven -especialmente sus frecuentaciones- tienen una influencia en su actividad delictiva.

La única diferencia significativa que se observa (Tabla7) entre los delincuentes especializados y versátiles de sexo femenino en términos de frecuentación de pares se encuentra en las niñas pertenecientes a un grupo delincuente. En efecto, el 62,5\% de las delincuentes versátiles contra $41,9 \%$ de las especializadas hace parte de un grupo de delincuentes $(0,01<p<=0,05)$. En los varones, también se encuentran diferencias en los individualistas asi como en los jovenes que pertenecen a un grupo no delincuente.

Tabla 7. Grupo de pares y diversidad

\begin{tabular}{|l|c|c|c|c|}
\hline & Individualistas & Grupo no-delincuentes & Grupo delincuentes & \\
\hline Niñas & & & & \\
\hline Dél. especializas (N=267) & $25.5 \%(\mathrm{n}=68)$ & $32.6 \%(\mathrm{n}=87)$ & $41.9 \%(\mathrm{n}=112)$ & $100 \%$ \\
\hline Dél. versatiles $(\mathrm{N}=32)$ & $18.8 \%(\mathrm{n}=6)$ & $18.8 \%(\mathrm{n}=6)$ & $62.5 \%(\mathrm{n}=20)$ & $100 \%$ \\
\hline Test de proporción & $\mathrm{Z}=0.90$ & $\mathrm{Z}=1.85$ & $\mathrm{Z}=2.27$ & \\
\hline Nivel de significación & $\mathrm{p}>0.10$ & $0.05<\mathrm{p}<=0.10$ & $0.10<\mathrm{p}<=0.05$ & \\
\hline Varones & & & & $41.8 \%(\mathrm{n}=173)$ \\
\hline Dél. especializados $(\mathrm{N}=414)$ & $27.5 \%(\mathrm{n}=114)$ & $30.7 \%(\mathrm{n}=127)$ & $100 \%$ \\
\hline Dél. versatiles (N=113) & $11.5 \%(\mathrm{n}=13)$ & $11.5 \%(\mathrm{n}=13)$ & $77.0 \%(\mathrm{n}=87)$ & $100 \%$ \\
\hline Test de proporción & $\mathrm{Z}=4.30$ & $\mathrm{Z}=5.10$ & $\mathrm{Z}=7.58$ & \\
\hline Nivel de significación & $\mathrm{p}<=0.001$ & $\mathrm{p}<=0.001$ & $\mathrm{p}<=0.001$ & \\
\hline
\end{tabular}


Para las dos categorías de la variable, hay significativamente más delincuentes especializados haciendo parte de los individualistas $(27,5 \%)$ o teniendo un grupo de amigos no delincuentes $(30,7 \%)$ que los delincuentes versátiles (respectivamente $11,5 \%$ y $11,5 \%$ ). Esto para el mismo nivel de significación $(\mathrm{p}<=0,001)$.

En fin, en relación a la categoria "grupo de delincuentes», se encuentró la misma diferencia que en las niñas a saber que $77,0 \%$ de los delincuentes versátiles que frecuentan a un grupo de amigos delincuentes amigos mientras que sólo el 41,8\%oncernidos ( $\mathrm{p}<=0,001$ ).

Los análisis muestran que de manera general, los jóvenes infractores de la muestra de la ISRD-2 no están distribuidos de manera aleatoria en términos de frecuentación de pares (Tabla 7.). Los individuos con una delincuencia versátil tienen más tendencia de pertenecer a un grupo de jóvenes delincuentes.

Esta tabla también permite igualmente avanzar la idea de que hay una diferencia entre los géneros en términos de frecuentación de pares y esto, en particular en los delincuentes versátiles. Se observa que los varones con una delincuencia versátil estan ampliamente poco representados en las dos primeras categorías de la variable (respectivamente $11,5 \%$ y $11,5 \%$ ), entonces son una gran proporción $(77,0 \%)$ la que pertenece a un grupo delincuente. Las delincuentes versátiles son más numerosas que los varo- nes a formar parte de las dos primeras categorías de la variable independiente (respectivamente 18,8\% y 18,8\%) y se encuentran en menor número $(62,5 \%)$ que los varones en la categoría «grupo delincuente» .

En los individuos con una delincuencia especializada, el efecto del género no parece tener un impacto sobre el tipo de frecuentación que adoptan los adolescentes. En efecto, hombres y mujeres pertenecen en la misma proporción a las tres categorías de la variable.

\subsection{Perfiles delincuentes y la teoría de la elección racional}

Se analiza ahora los dos grupos de delincuentes para ver si ellos difieren a la luz de la teoría de la elección racional (Scott, 2000; Cornish y Clarke, 1985). Este último establece que la implicación en una actividad delictiva versátil o especializada depende de los valores, las creencias, los códigos morales del individuo y su tendencia a tolerar la violencia como siendo un modo adecuado o no de resolución de conflictos.

Los resultados (Tabla 8) obtenidos después del análisis de la población femenina muestra que la única diferencia entre los dos grupos de delincuentes reside en la categoria "baja tolerancia a la violencia". Hay significativamente más delincuentes especializadas $(24,7 \%)$ que delincuentes versátiles $(9,4 \%)$ que tienen una baja tolerancia a la violencia $(0,001<\mathrm{p}<=0,01)$.

Tabla 8. Tolérancia hacia la violencia y la diversidad

\begin{tabular}{|l|c|c|c|c|}
\hline & Tolerancia debil & Tolerancia media & Tolerancia fuerte & \\
\hline Niñas & & & & \\
\hline Dél. especializada (N=263) & $24.7 \%(\mathrm{n}=65)$ & $8.0 \%(\mathrm{n}=21)$ & $67.3 \%(\mathrm{n}=177)$ & $100 \%$ \\
\hline Dél. versatiles $(\mathrm{N}=32)$ & $9.4 \%(\mathrm{n}=3)$ & $9.4 \%(\mathrm{n}=3)$ & $81.3 \%(\mathrm{n}=26)$ & $100 \%$ \\
\hline Non-delincuentes $(\mathrm{N}=1365)$ & $1.8 \%(\mathrm{n}=25)$ & $43.0 \%(\mathrm{n}=587)$ & $55.2 \%(587)$ & $100 \%$ \\
\hline Test de proporción & $\mathrm{Z}=2.63$ & $\mathrm{Z}=0.26$ & $\mathrm{Z}=1.87$ & \\
\hline Nivel de significación & $0.001<\mathrm{p}<=0.01$ & $\mathrm{p}>0.10$ & $0.05<\mathrm{p}<=0.10$ & \\
\hline Varones & & & & $77.9 \%(\mathrm{n}=318)$ \\
\hline Dél. spécialiazos $(\mathrm{N}=408)$ & $18.4 \%(\mathrm{n}=75)$ & $3.7 \%(\mathrm{n}=15)$ & $100 \%$ \\
\hline Dél. versatiles $(\mathrm{N}=112)$ & $2.7 \%(\mathrm{n}=3)$ & $1.8 \%(\mathrm{n}=2)$ & $95.5 \%(\mathrm{n}=107)$ & $100 \%$ \\
\hline No-delincuentes $(\mathrm{N}=1014)$ & $6.6 \%(\mathrm{n}=67)$ & $55.3 \%(\mathrm{n}=561)$ & $38.1 \%(\mathrm{n}=386)$ & $100 \%$ \\
\hline Test de proporción & $\mathrm{Z}=6.39$ & $\mathrm{Z}=1.21$ & $\mathrm{Z}=6.20$ & \\
\hline Nivel de significacion & $\mathrm{p}<=0.001$ & $\mathrm{p}>0.10$ & $\mathrm{p}<=0.001$ & \\
\hline
\end{tabular}


Referente a la categoría «alta tolerancia hacia la violencia», los dos grupos de delincuentes de sexo femenino presentan en las mismas proporciones un alto porcentaje de personas con una alta tolerancia hacia la violencia.

En los varones, la misma diferencia que en las niñas se encuentran en la categoría «baja tolerancia a la violencia». Los delincuentes especializados presentan 18,4\% contra solamente $2,7 \%$ de los delincuentes versátiles perteneciendo a esta categoría ( $\mathrm{p}<=0,001)$.

Sin embargo, se observa una diferencia entre los dos grupos de interés en la última categoría de la variable. En efecto, hay significativamente más delincuentes versátiles $(95,5 \%)$ que delincuentes especialisados $(77,9 \%)$ con una alta tolerancia a la violencia $(\mathrm{p}<=0,001)$.

Analizando las diferencias entre varones versus niñas de la población delincuente, se ve que, independientemente de su tipo de delincuencia, las niñas son más numerosas que los varones a tener una tolerancia de débil a mediana a la violencia, mientras que los chicos se encuentran en mayor número en la categoría «alta tolerancia».

Sin embargo, y en contra de todas las expectativas, estas diferencias de género se invierten en la población de no delincuente. Las niñas (32,7\%) son menos numerosas que los varones $(22,1 \%)$ a tener una tolerancia de baja a media. En general, los resultados obtenidos concerniente la variable "tolerancia hacia la violencia» se deben matizar. Efectivamente, una gran cantidad de personas, sean delincuentes o no tienen una alta tolerancia hacia la violencia. Esto es pues común tanto entre los delincuentes como en los no delincuentes. Notese que estos resultados muy elevados en la población no infractora se desprenden de una metodología discutible en términos de la creación de la variable. Las preguntas de la encuesta utilizadas para la creación de la variable «tolerancia hacia la violencia» no son necesariamente adecuadas para probar las hipótesis de este estudio-

\section{Discusión / Conclusión}

Las diferentes teorías planteadas por la literatura criminológica que trata del tema objeto de este estudio han buscado pruebar sus hipótesis en poblaciones particulares. Estas se componen de individuos delincuentes y conocidos por las autoridades, algunos son recidivistas. En el presente estudio, se trata de jóvenes escolarizados y no conocidos por las autoridad y la mayoría no presentan los perfiles de una delincuencia seria, pero sus comportamientos reflejan las experiencias de aprendizaje social que a menudo son un reflejo de este difícil período que es la adolescencia en la vida del hombre.
Vease ahora más en detalle el análisis a la luz de las cuatro hipótesis propuestas.

1. La primera hipótesis aborda las teorías del desarrollo compara los delincuentes especializados y los delincuentes versátiles desde el punto de vista de tres factores: la edad de inicio en la delincuencia, la incidencia de la comisión de los delitos asi como la violencia de los delitos cometidos. En primer lugar, en relación con la edad de inicio, se esperaba que los delincuentes versátiles se iniciaran en la actividad criminal en promedio a una edad más temprana que los delincuentes especializados. Sin embargo, los resultados del análisis en este estudio no apoyan esta afirmación, de hecho, los delincuentes versátiles de la ISRD-2 no cometen sus primeros delitos a una edad más temprana que los delincuentes especializados. Sin embargo, se nota que la escala utilizada puede ser la causa de esta falta de diferencia estadística. Se eligió 12 años como el límite para considerar a un delincuente precoz. Este límite de edad se inspira en la literatura criminológica que estudia con frecuencia jóvenes muy implicados en la delincuencia y a menudo conocidos de las autoridades judiciales. La población sobre la que se realizó estos análisis es una población compuesta por pocos delincuentes serios, esto es por eso que el límite de 12 años probablemente se debio aumentar unos años para observar una diferencia estadística. Sin embargo, si se hubiera hecho, habría otro problema: los jóvenes de la ISRD-2 tienen entre 13 y 16 años, aumentanto el limite de edad algunos datos pueden haberse perdido.

Los dos últimos elementos de la hipótesis 1 relativos a la frecuencia con la que una persona comete delitos y la gravedad de éstos. La hipótesis planteada en la literatura criminológica es que los delincuentes versátiles cometen más delitos y una mayor frecuencia mayor que los delincuentes especializados. Según los análisis de la ISRD-2, los resultados obtenidos corroboran estas proposiciones. La frecuencia de comisión de los delitos asi como su gravedad se producen no sólo en los delincuentes versátiles, sino también más específicamente en los varones. Las chicas, tengan una delincuencia versátil o especializada cometen delitos a una más baja frecuencia y con un carácter menos grave que los varones. Estos resultados confirman el hecho de que, incluso si una niña está muy involucrada en la delincuencia, su actividad criminal es menos frecuente y menos orientada hacia la violencia de lo que es para los varones. En efecto, algunos estudios (Tremblay, 2000) muestran que si la agresión física de los varones tiende a aumentar con la edad y la participación en la delincuencia, la de las niñas, 
tiende a disminuir para dar paso a una violencia más de tipo psicológica.

2. Hipótesis 2 sugiere que los delincuentes versátiles tienen un nivel de autocontrol más bajo que los delincuentes especialisados. Los resultados obtenidos del análisis son consistentes con esta hipótesis. De hecho, hay más delincuentes versátiles que delincuentes especializados que presentan un nivel de autocontrol bajo. Al contrario, no se encontró ninguna diferencia estadística entre los géneros. De acuerdo con los datos de la ISRD-2, entre los delincuentes los varones como las niñas tienen una proporción similar de personas con un bajo nivel de autocontrol. Esto sugiere que este rasgo de la personalidad no es el resultado del sexo de la persona, ya que se distribuye por igual en la población. Aunque esto, sale de las preguntas problemas de esta investigación, habría sido interesante probar la diferencia de proporción de individuos presentando un bajo nivel de autocontrol entre los delincuentes y los no delincuentes. Esto con el fin de comprobar si la presencia de este rasgo de la personalidad lleva a un comportamiento delictivo.

3. La tercera hipótesis propuesta es que los jóvenes que frecuentan un grupo de jóvenes delincuentes se implican en una delincuencia más versátil que los jóvenes que tienen tendencia a frecuentar amigos no delincuente o no premanecen solos (los individualistas). Los resultados muestran una mayor proporción de delincuentes versátiles que pertenecen a grupos de jóvenes delincuentes. Y a la inversa, los delincuentes especializados están más ampliamente representados en los grupos de jóvenes no delincuentes y en la categoría de jóvenes que no frecuentan ningún grupo. Parecería bien que el hecho de frecuentar otros delincuentes engendra a menudo una influencia mutua y un aprendizaje social que lleva al individuo a cometer delitos muy diferentes unos de otros. Sin embargo, se tiene reserva sobre la generalización de estas consideraciones. Porque no se puede saber la dirección de la relación: ¿es debido al hecho de que el individuo frecuente a un grupo que él se implica en una delincuencia versátil, o es porque él comete delincuencia versátil que él tiende a frecuentar grupos de jóvenes con las mismas tendencias? Lamentablemente, sólo un estudio longitudinal seria susceptible de aportar elementos de respuestas.

4. Finalmente, la última hipótesis que se quizo probar anuncia que los individuos versátiles tienen creencias, valores y códigos morales tolerando más la delincuencia-la violencia-que los individuos especializados. Se observa aqui una diferencia estadística entre los delincuentes versátiles y especializados. Los versátiles son de hecho más numerosos a tener un valores fuertemente enfocados hacia la delincuencia violenta, mientras que los delincuentes especializado, son más numerosos a tener valores morales tolerando medianamente o más bien ligeramente delincuentes los actos delictivos violentos. Los mismos resultados se observaron entre los varones y las niñas. Los muchachos tienen valores fuertes mientras que las niñas tienden a valores medianos o debil. Sin embargo, se debe precisar que esta variable tiene un sesgo operacional importante que puede sesgar los resultados en términos de diferencia varones-niñas. Las preguntas utilizadas para crear esta variable «valores delincuentes» se basan en conceptos que se focalizan más en los varones que en las niñas. Se puede citar aquí la pregunta: «Es totalmente normal que se quiera mostrar que se un hombre peleandose con los demás.» Se puede fácilmente concluir que las niñas entrevistados se sentían poco concernidas por esta pregunta.

\section{Conclusión}

Se puede concluir este trabajo afirmando que, dado el carácter cuantitativo del mismo y su enfoque puramente estadístico, bastantes elementos de la población estudiada seguirá siendo un misterio. No se cuestiona modo alguno la capacidad que tienen las estadísticas de aprehender el más grande número de personas a fin de categorizarlas y de conocer las propiedades de cada grupo distinto en una población tan heterogenea sea ella. Se admira igualmente todo el valor científico que ellas tienen, se esta conscientes de cómo los números hablan a menudo mucho mejor y mucho más explícitos que las palabras.

Sin embargo, se lamenta a veces la frialdad de este enfoque, que tiene por objeto, en cierta forma, una matematización del espíritu humano y su comportamiento. A lo largo de este estudio, se ha sentido a veces una especie de tortura por la imposibilidad de no poder entrar en contacto con estos jóvenes y poder preguntarles, para saber más de dónde vienen, cómo funcionan y a dónde se dirigen. En lugar de eso, se ha estado ante cuadrículas analíticas que muestran las tendencias generales de cada grupo y sus generalizaciones. Se creemos en lo enriquecedor que seria el pasaje de lo general a lo individuo. Esta reciprocidad entre los enfoques cualitativos y cuantitativos permite ir, más allá de lo meramente numérico y ser capaz de comprender una problematica en su conjunto. 


\section{Referencias}

Aebi, M. F. (2006). Comment mesurer la délinquance. Paris : Armand Colin.

Armstrong, T.A. (2008). Exploring the impact of changes in group composition on trends in specialization. Crime $\mathcal{E}$ Delinquency, 54 (3), 366-389.

Armstrong, T.A., \& Britt, C. L. (2004). The effect of offender characteristics on offense specialization and escalation. Justice Quarterly, 21(4), 843-76.

Blumstein, A., Cohen, J., Roth, J.A., \& Visher, C.A. (1986). Criminal careers and "career criminals" 1. Washington, DC: National Academy Press.

Cohen, L.E., \& Felson, M. (1979). Social change and crime rate trends: A routine activity approach. American sociological Review, 44(4), 588-608.

Cornish, D.B., \& Clarke, R.V. (1985). Modeling offenders'descisions: A framework for research and policy. In M.H. Tonry, \& N. Morris (Eds.), Crime and justice: An annual review of research (pp.147-185). Chicago:university of Chicago press.

Dean, C.W., Brame, R., \& Piquero, A. (1996). Criminal propensities, discrete groups of offenders, and persistence in crime. Criminology, 34(4), 547-570.

Farrington, D.P. (1986). Age and crime. In M.H. Tonry, \& N. Morris (Eds.), Crime and Justice:A Review of Research,7 (pp. 189-250). Chicago: University of Chicago Press.

Farrington, D.P., Snyder, H.N., \& Finnegan, T.A. (1988). Specialization in juvenile court careers. Criminology, 26, 461-487.

Farrington, D.P. (2005). The integrated cognitive antisocial potential (ICAP) theory. In David P. Farrington. (Eds.), Advances in Criminological Theory. Integrated Developmental \& Life-course Theory of Offending, 14 (pp. 57-67). New Brunswick, NJ: Transaction Publishers.

Guerette, R.T., Stenius, V.M.K., \& McGloin, J.M. (2005). Understanding offense specialization and versatility: A reapplication of the rational choice perspective. Journal of Criminal Justice, 33(1), 77-87.

Gottfredson, M.R. (2005). Offender classifications and treatment effects in developmental criminology: A propensity/ event consideration. Annals of the American Academy of Political and Social Science, 602, 46-57.

Gottfredson, M.R., \& Hirschi, T. (1989). A propensity-event theory of crime. In W.S. Laufer, \& F. Adler. (Eds.), Advances in Criminological Theory, 1, (pp. 57-67). New Brunswick, NJ: Transaction Publishers.

Gottfredson, M.R., \& Hirschi, T. (1990). A general theory of crime. Stanford: Stanford University Press.

Gottfredson, M.R., \& Hirschi, T. (1993). Commentary: Testing the general theory of crime. Journal of research in crime and delinquency,30 (1), 47-54.

Gottfredson, M.R., \& Hirschi, T. (2003). Self-control and opportunity. In C.L. Britt, \& M.R. Gottfredson. (Eds.), Advances in Criminological Theory, 12, (pp. 5-19). New Brunswick, NJ: Transaction Publishers.

Killias, M., Aebi, M. F., Herrmann, L.; Dilitz, C., \& Lucia, S. (2009). Switzerland. In D. Enzmann, B. Gruszeynska, I. Haen-Marshal, J. Junger-Tas, M. Killias, \& M. Steketee (Eds.), Juvenile Delinquency in Europe and Beyond: an International Perspective on Key Issues and Causes. Springer.

Kempf, K.L. (1987). Specialization and the criminal career. Criminology, 25(2), 399-417.

Klein, M.W. (1984). Offence specialization and versatility among juveniles". British Journal of Criminology, Delinquency and Deviant Social Behaviour, 24 (2), 185-194.

Klein, M.W., \& Crawford, L.Y. (1967). Groups, gangs and cohesiveness. Journal of Research in Crime and Delinquancy, 4(1), 63-75.

Mason, W.A., \& Windle, M. (2002). Gendre, self-control, and informal social control in adolescence. A test of tree models of the continuity of delinquent behavior. Youth and Society, 33 (4), 479-514.

Mazerolle, P., Brame, R., Paternoster, R., Piquero, A., \& Dean, C.W. (2000). Onset age, persistence, and offending versatility: comparaison across gender. Criminology, 38(4), 1143-1171. 
McGloin, J.M., Sullivan, C.J., Piquero, A.R., \& Pratt, T.C. (2007). Local life circumstances and offending specialization/ versatility, comparing opportunity and propensity models. Journal of Research, 44(3), 321-346.

Moffitt, T.E. (1993). Life-course persistent and adolescent-limited antisocial behavior: A developmental taxonomy. Psychological Review, 100, 674-701.

Moffitt, T.E. (1997). Adolescent-limited and life-course persistent offending: A complementary pair of developmental theories. In T.P. Thornberry. (Eds.), Developmental Theories of Crime and Delinquancy (pp. 11-54). New Brunswick, NJ: Transaction.

Monahan, Z.A., \& Piquero, A.R. (2009). Investigating the longitudinal relation between offending frequancy and offending variety. Criminal Justice and Behavior,36 (7), 653-673.

Paternoster, R., Brame, R, Piquero, A., Mazerolle, P., \& Dean, C.W. (1998). The forward specialization coefficient: distributional properties and subgroup differences. Journal of Quantitative Criminology, 14(2), 133-154.

Peretti, P. (2001-3). Théories de la Déviance et Délinquance Auto-reportée en Milieu Scolaire. Déviance et Société, 25, 23556.

Piquero, A., Paternoster, R., Mazerolle, P., Brame, R., \& Dean, C. W. (1999). Onset Age and Offense Specialization. Journal of Research in Crime and Delinquency, 36(3), 275-299.

Piquero, A. (2000). Frequency, specialization and violence in offending careers. Journal of Research in Crime and Delinquency,30 (4), 392-418.

Reiss, A.J. (1986). Co-offender influences on criminal careers. In A. Blumstein, J. Cohen, J. Roth, \& C. Visher (Eds.), Criminal Careers and "Career Criminals",2 (pp. 121-157). Washington D.C.: National Academy Press.

Bursik, R.J. (1980). The dynamics of specialization in juvenile offenses. Social Forces,58(3), 851-64.

Scott, J. (2000). Rational choice theory. In G. Browning, A. Haldi, \& F. Webster (Eds.), Understanding contempory society: Theories of the Present (pp. 126-139). Londres: Sage Publication.

Tremblay, R. E. (2000). L'origine de la violence chez les jeunes. Isuma, 1(2), 19-24.

Warr, M. (1996). Organization and instigation in delinquent groups". Criminology, 34(1), 11-37. 